\title{
Principles for reducing the phase shift between instantaneous analogue and discrete signals and the corresponding values in the sampled values (SV) stream of a digital substation
}

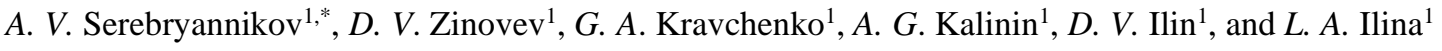 \\ ${ }^{1}$ I.N. Ulyanov Chuvash State University, Cheboksary, Russia
}

\begin{abstract}
There are numerous publicly available articles on digital substations, including those dedicated to current and voltage measurements, time synchronization, test systems development, principles of substation design, and other aspects of this topic. But the phase shift between instantaneous signals and the corresponding values in the SV (sampled values) stream has been little discussed. We can list the following principles to reduce the phase shift between instantaneous analogue and discrete signals and the corresponding values in the SV stream. 1) Exclusion from the device, of non-essential elements that introduce additional propagation delays of analogue and discrete signals, provided that such exclusion does not adversely affect the device performance or characteristics. 2) Reduction of the delay time for the other device elements to the minimum values. 3) Adjustment of the delay time of some device elements or addition of special delay elements to equalize the time of signal propagation over analogue and discrete channels. 4) Consideration of the total delay time of signal propagation over analogue and discrete channels and adjustment of the time stamp in the SV frame. The article provides examples of application of these principles in signal generation and measuring devices and phase shift meters.
\end{abstract}

\section{Principles for reducing the phase shift between instantaneous analogue and discrete signals and the corresponding values in the SV stream}

There are numerous publicly available articles on digital substations, including those dedicated to current and voltage measurement [1-5], time synchronization [6-10], test systems development [11-12], principles of substation design [13-14], and other aspects of this topic [15-20]. But the phase shift between instantaneous signals and the corresponding values in the SV (sampled values) stream has been little discussed.

We can list the following principles for reducing the phase shift between instantaneous analogue and discrete signals and the corresponding values in a SV stream.

1. Exclusion from the device, of non-essential elements that introduce additional propagation delays of analogue and discrete signals, provided that such exclusion does not adversely affect the device performance or characteristics.

2. Reduction of the delay time for the other device elements to the minimum values.

3. Adjustment of the delay time of some device elements or addition of special delay elements to equalize the time of signal propagation over the analogue and discrete channels.
4. Consideration of the total delay time of signal propagation over the analogue and discrete channels and adjustment of the time stamp in the SV frame.

\section{An example of application of the principles in a signal generation device and a phase shift meter}

Figure 1 shows an example of a joint operation of a signal generator and a phase shift meter. These devices must be synchronized with the master clock (time server), for example, via the PTP protocol. This protocol ensures that the following relations will be satisfied:

$$
\begin{aligned}
& \left|T_{\text {cur } 1}-T_{\text {cur } 2}\right| \leq 1 \mathrm{~ms}, \\
& \left|T_{\text {cur }}-T_{\text {cur } 1}\right| \leq 1 \mathrm{~ms}, \\
& \left|T_{\text {cur }}-T_{\text {cur } 2}\right| \leq 1 \mathrm{~ms}
\end{aligned}
$$

$T_{\text {cur }}$ is the current time of the time server (exact time), $T_{\text {cur1 }}$ and $T_{\text {cur2 }}$ are the current times of the first and the second devices.

The external computer sets the parameters of the signals generated by the first device. The internal computer generates a digital signal according to the task. This signal is amplified to the required level to be transmitted over a digital line. For transmission over an analogue line, the original digital signal must pass through a digital-to-analogue converter (DAC) and a

*Corresponding author: alex-silver@mail.ru 


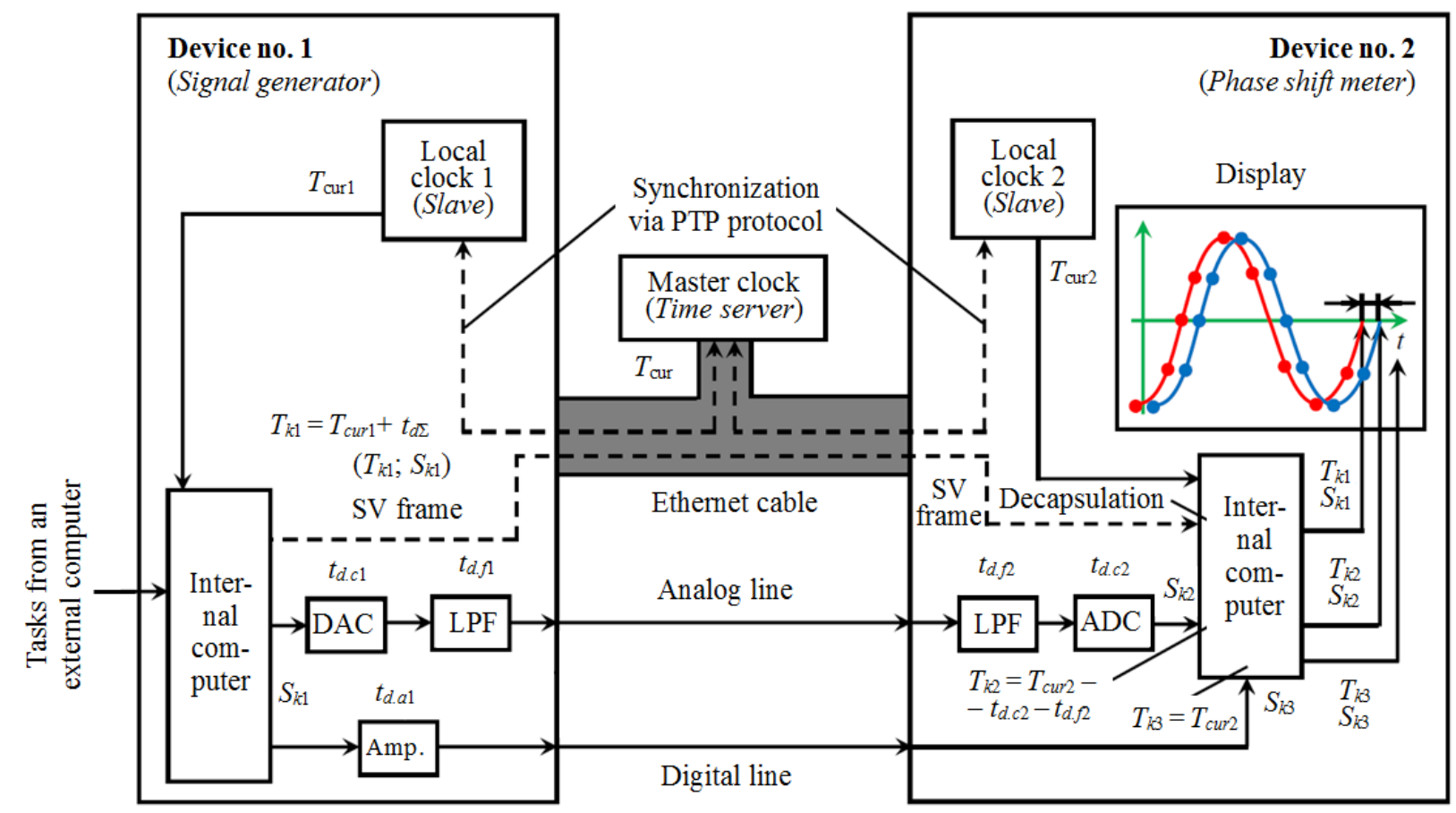

Fig. 1. An example of a joint operation of a signal generator and a phase shift meter.

low-pass filter (LPF). If necessary, the received signal must also be passed through the amplifier.

It is necessary to minimize the difference between the total delays in the analogue and digital channels using the above principles in order to satisfy the condition:

$$
t_{\text {d.a1 }} \approx t_{d . c 1}+t_{d . f 1}=t_{d \Sigma} .
$$

$t_{d . a 1}$ is the delay time of the amplifier in the digital channel, $t_{d . c 1}$ and $t_{d . f 1}$ are the delay times of the converter and the filter in the analogue channel, $t_{d \Sigma}$ is the indication of the total delay in these channels.

The time delays can be calculated or measured at the device design stage, and the total time delays in the analogue and digital channels $t_{d \Sigma}$ can be made approximately the same. When forming the SV frame, this delay can be taken into account at time stamping

$$
T_{k 1}=T_{c u r 1}+t_{d \Sigma} \text {. }
$$

$T_{k 1}$ is the time value in the $\mathrm{SV}$ frame corresponding to the sample $S_{k 1}$.

The described measures will allow reduction of the phase shifts between the analogue and digital signals and the signal values in the SV stream.

In the phase shift meter (in the second device), the $\mathrm{SV}$ frame undergoes decapsulation in the internal computer, so the current sample will have the same time value $T_{k 1}$ as set for the first device. The digital signal to the internal computer arrives without delay. In the analogue channel, there are delays $t_{d . f 2}$ and $t_{d . c 2}$ in the low-pass filter and the analogue-to-digital converter (ADC). The internal computer must take these delays into account and adjust the time value $T_{k 2}$ for the digitized value:

$$
T_{k 2}=T_{c u r 2}-t_{d . c 2}-t_{d . f 2} .
$$

After the adjustment, all the three signals can be displayed on the screen, for their phase shifts to be compared. The internal computer can also calculate these phase shifts and show them on the display, because they may be otherwise unnoticeable.

\section{An example of application of the principles in a measuring device}

In the signal meter (Figure 2), additional delays may appear in the current transformer $t_{d . c t 1}$ and the voltage transformer $t_{d . v t 1}$. It is desirable that the following conditions be satisfied:

$$
t_{d . c t 1}=t_{d . v t 1}=t_{d . t 1} .
$$

If the delay times $t_{d . c t 1}$ and $t_{d . v t 1}$ are sufficiently small, they can also be neglected.

Over the analogue lines, signals propagate without delays (unless delays in the transformers are taken in account). It is necessary to minimize delays in the digital channels in the low-pass filter $t_{d . f 1}$ and the ADC $t_{d . c 1}$, or introduce additional delays in the analogue channels to satisfy the condition:

$$
t_{d \Sigma a} \approx t_{d \Sigma d}=t_{d \Sigma} .
$$

$t_{d \Sigma a}$ and $t_{d \Sigma d}$ are the total delays in the analogue and digital channels. For the case shown in Figure 2

$$
t_{d \Sigma a}=t_{d . t 1}, t_{d \Sigma d}=t_{d . t 1}+t_{d . f 1}+t_{d . c 1}+t_{d . a 1} .
$$

When forming an SV frame, the internal computer must consider this delay at time stamping: 


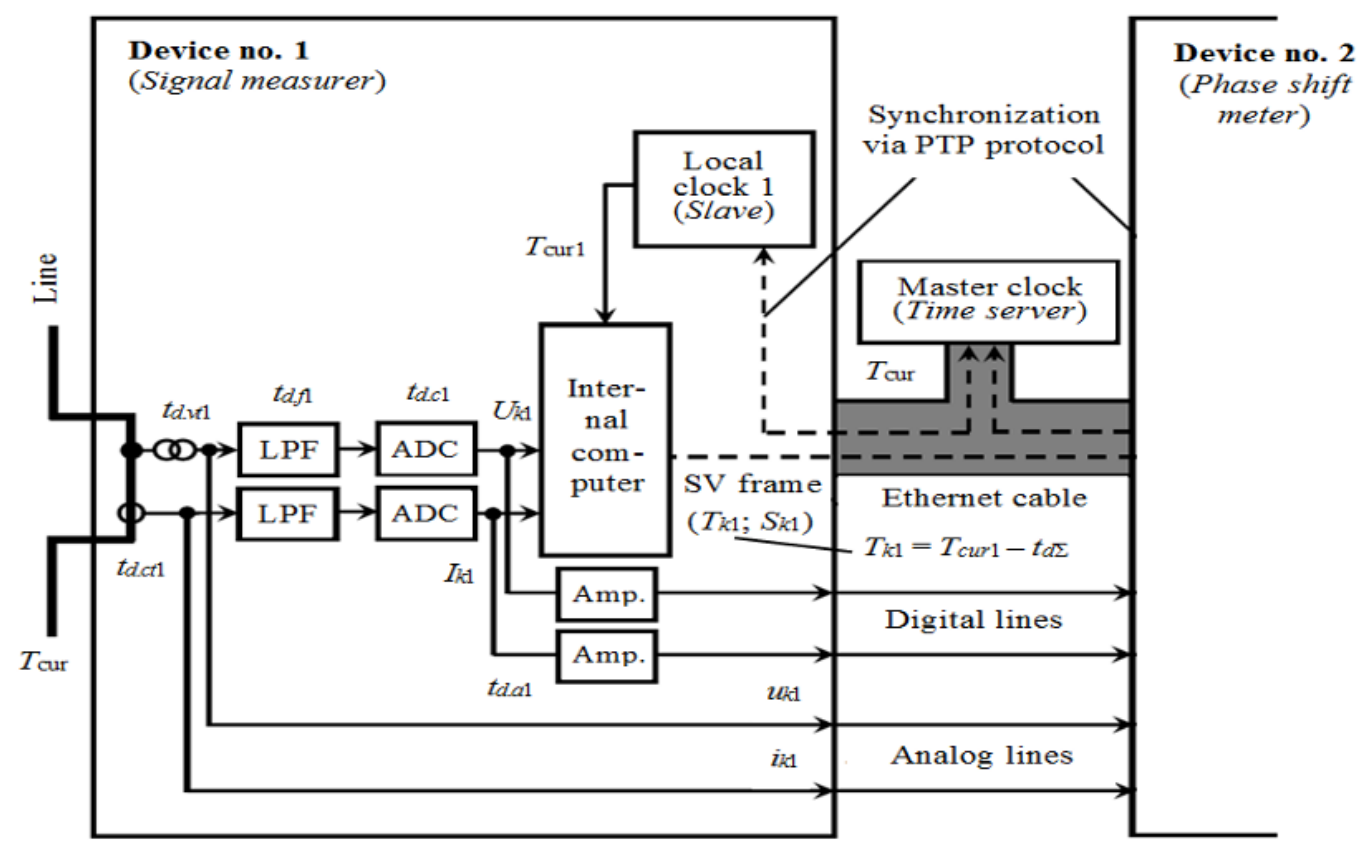

Fig. 2. The structure of the signal meter.

$$
T_{k 1}=T_{c u r 1}-t_{d \Sigma}
$$

As you can see, the $t_{d \Sigma}$ value needs to be subtracted from the current time value $T_{\text {cur } 1}$.

\section{Conclusions}

This article lists the basic principles for reducing the phase shift between instantaneous analogue and discrete signals and the corresponding values in a SV stream in a digital substation. Two examples illustrate the practical application of these principles.

The structural diagrams of the generator and the signal meter, as well as the phase shift meter shown in the examples, can be very useful in designing the circuits of these devices. The formulas given in this article can be used in the algorithms of the internal computers of the said devices to reduce the phase shift between instantaneous analogue and discrete signals and the corresponding values in the SV stream.

\section{References}

[1] W.J. Ma, L.H. Mu, P. Ao, X. Zhang, New type merging unit for digital substation Conf. on Advanced Power System Automation Protection 1598601 (2011)

[2] Y. Wang, Y. Zhang, J. Tang, Study of multisample-rate signal conversion in digital substation and wide area measurement of power system Int. Conf. on Sustainable Power Generation and Supply 1-4 (2009)

[3] J. Rong, G.-X. Zhang, X.-M. Zhu, P. Wang, Electric energy measurement in digital substation on a number of issues discussed China Int. Conf. on Electricity Distribution 1-5 (2008)
[4] Z. Feng, C. Yingying, X. Ji, D. Jie, Research of digital metering system and calibration technology of smart substation IEEE Workshop on Advanced Research and Technology in Industry Applications 1240-42 (2014)

[5] D.V. Topolsky, I.G. Topolskaya, N.D. Topolsky, Intelligent instrument transformer for control systems of digital substations Ural Symp. on Biomedical Engineering, Radioelectronics and Information Technology 174-8 (2018)

[6] J. Hu, G. Xin-Hua, Application of time synchronization system based on SNTP in digital substation China Int. Conf. on Electricity Distribution 14 (2008)

[7] K. Wang, Y. Hu, H. Ma, Research and design on time synchronization technology of smart substation based on IEEE1588 Int. Conf. on Advanced Power System Automation and Protection 2244-48 (2011)

[8] R. Moore, R. Midence, M. Goraj, Practical experience with IEEE 1588 high Precision Time Synchronization in electrical substation based on IEC 61850 Process Bus Proc. of 2010 IEEE PES General Meeting 1-4 (2010)

[9] K. Zhang, D. Zhang, Z. Deng, Y. Zhao, J. Li, L. Zhang, Intelligent substation time synchronization device information model based on IEC Int. Conf. on Advanced Power System Automation and Protection 2218-22 (2011)

[10] H. Zhao, X. Wang, Z. Yang, Design and implementation of precision time synchronization system based on IEEE1588 4th Int. Conf. on Electric Utility Deregulation and Restructuring and Power Technologies 610-3 (2011)

[11] M. Kai, H. Shu, C. Jiongcong, Y. Yingan, Discussion of the testing technology for protection 
device used in digital substation China Int. Conf. on Electricity Distribution 1-5 (2010)

[12] B. Tang, X. Li, X. Yang, H. Fan, Development of dynamic test system for digital relay protection based on IEC61850 standards Int. Conf. on Advanced Power System Automation and Protection 1594-97 (2011)

[13] Q. Fu, J. Chen, Design of experiment platform for digital substation based on IEC 61850 5th Int. Conf. on Computer Science and Network Technology 4-8 (2016)

[14] L. Siyuan, Z. Gao, Z. Yi, W. Tao, R. Mu, Research on designing methods of the secondary system in digital substation IEEE PES Asia-Pacific Power and Energy Engineering Conf. 289-93 (2016)

[15] X.G. Chen, Y.T. Qiu, J.N. Jiang, B.Q. Zhu, W.L. Pan, Y.J. Yang, A new fault recording system based on spectroscope for intelligent substation Int. Conf. on Advanced Power System Automation and Protection 470-3 (2011)

[16] A. Wuthayavanich, K. Santayanon, R. Rujitanyatarn, M. Rakpan, T. Bhothigun, Future Approach and Advantages of IEC 61850 Implemented for EGAT Digital Substation IEEE PES GTD Grand Int. Conf. and Exposition Asia 446-9 (2019)

[17] M. Schumacher, C. Hoga, J. Schmid, Get on the digital bus to substation automation IEEE Power and Energy Magazine 5(3), 51-6 (2007)

[18] A.G. Merkulov, R. Adelseck, J. Buerger, Wideband digital power line carrier with packet switching for high voltage digital substations IEEE Int. Symp. on Power Line Communications and Its Applications 1-5 (2018)

[19] L. Zhu, N. Dong, X. Duan, J. Chen, D. Shi, Research on Digital Simulation Platform for Networked Substation IEEE Power Engineering Society General Meeting 1-4 (2007)

[20] D.V. Topolskiy, I.G. Topolskaya, E.A. Sirotkin, Improvement of efficiency of information exchange between a digital substation and a grid control centre Int. Conf. on Industrial Engineering, Applications and Manufacturing 1-6 (2017) 